\title{
Pathological Correlates of Extrapyramidal Signs in Alzheimer's Disease
}

\author{
Yan Liu, MD, MS, ${ }^{*}$ Yaakov Stern, PhD, $\dagger$ Michael R. Chun, MD, $\ddagger$ Diane M. Jacobs, PhD, $\dagger$ \\ Patricia Yau,* and James E. Goldman, MD, PhD*
}

Extrapyramidal signs frequently accompany Alzheimer's disease (AD), but the pathological substrate remains unknown. Clinical and postmortem information from patients with $\mathrm{AD}$, Parkinson's disease, or progressive supranuclear palsy and control subjects seen at a large tertiary medical center between 1989 and 1994 was examined. AD patients who had taken neuroleptics and $\mathrm{AD}$ brains that also contained Lewy bodies were excluded. The presence of extrapyramidal signs was determined using the Unified Parkinson's Disease Rating Scale. Sections of basal ganglia, subthalamic nucleus, and substantia nigra were examined for neurofibrillary tangles and neuropil threads and the nigra for neuronal numbers. Patients with AD (with or without extrapyramidal signs) did not show neuronal loss in the nigra compared to control subjects, while both Parkinson's disease and progressive supranuclear palsy brains showed marked depletion. The number of neurofibrillary tangles and neuropil threads was increased in AD (with or without extrapyramidal signs) nigra compared to control tissue, and also in progressive supranuclear palsy nigra, but not Parkinson's disease nigra. The numbers of nigral neurofibrillary tangles and neuropil threads were positively related to extrapyramidal signs in $\mathrm{AD}$. There were no correlations between tangles and threads in the basal ganglia or subthalamic nucleus and extrapyramidal signs in AD. Thus, extrapyramidal signs in AD correlate best with tangle pathology in the nigra and do not require the concomitant presence of Lewy bodies.

Liu Y, Stern Y, Chun MR, Jacobs DM, Yau P, Goldman JE. Pathological correlates of extrapyramidal signs in Alzheimer's disease. Ann Neurol 1997;41:368-374

Extrapyramidal signs (EPSs) frequently accompany Alzheimer's disease (AD) [1-4] and their presence is related to more rapid cognitive and functional decline and earlier death [1]. The anatomical and pathological basis for EPSs in AD has not been clear, however. The possibility that EPSs may result from concomitant Parkinson's disease (PD) is suggested by the presence of Lewy bodies in the substantia nigra of many AD patients. For example, pathological analyses of $\mathrm{AD}$ series revealed Lewy body changes in 25 to $55 \%$ of $\mathrm{AD}$ patients [5-8]. The variation among studies is no doubt related in large part to differences in the specific patient populations examined.

Nevertheless, AD patients without Lewy bodies do manifest EPSs [8]. Studies of the neuropathology that could underlie EPSs in AD have been confounded by the treatment of many patients with neuroleptic medication, by only a qualitative analysis of nigral pathology, and by only cursory descriptions of nigral changes. Nigral neuropathology is indeed present in $A D$ in the form of neurofibrillary tangles (NFTs), as noted in sev- eral studies $[6,9,10]$, but has not been described in detail using a variety of sensitive detection methods. The numbers of nigral neurons, assessed either qualitatively $[2,4,8]$ or quantitatively [3], appear to be normal or near normal, with at most only a mild decrease, and thus represent a considerable difference from the marked neuronal depletion seen in PD. Furthermore, normal levels of tyrosine hydroxylase, dopamine $\mathrm{D}_{2}$ receptor, and dopamine transporter are found in the $\mathrm{AD}$ nigra [11], consistent with a normal or near-normal neuronal population. Despite this relarively normal neuronal component, there is a loss of dopamine transporter in the striata of individuals with $A D$ and parkinsonism [11]. This loss implies that there are important pathological changes in the nigrostriatal projections.

We reexamined the problem of EPSs in AD by examining the brains of patients with clinical and pathologically confirmed $A D$, some of whom manifested EPSs. To isolate AD changes from those of PD, we excluded $\mathrm{AD}$ patients with Lewy bodies from our anal-
From the ${ }^{*}$ Department of Pathology (Division of Neuropathology) and the †Departments of Neutology and Psychiatry and the Gertrude H. Sergievsky Center, Columbia University, College of Physicians and Surgeons, New York, NY, and the łEverett Neurological Center, Everett, WA.

Received May 30, 1996, and in revised form Aug 15. Accepted for publication Aug 23, 1996.
Address correspondence to Dr Liu, Department of Pathology (Division of Neuropathology), 630 West 168th Street, New York, NY 10032. 
ysis. We also compared the numbers of neuronal cells, NFTs, and neuropil threads (NPTs) in AD with those in $\mathrm{PD}$ and progressive supranuclear palsy (PSP).

\section{Research Design and Methods Subjects}

AD CASE SELECTION. Patients with a diagnosis of AD were selected from all autopsy reports at Columbia Presbyterian Medical Center from 1989 through 1994. We then examined neurological and neuropsychological clinical records to evaluate the presence or absence of EPSs. The inclusion and exclusion criteria for $\mathrm{AD}$ cases were as follows:

All included individuals had been examined at Columbia by members of the neurology staff. Each subject met $\mathrm{Na}$ tional Institute of Neurological and Communicative Disorders and Stroke-Alzheimer's Disease and Related Disorders Association (NINCDS-ADRDA) criteria for probable AD during life [12], EPSs must have been assessed using the Unified Parkinson's Disease Rating Scale (UPDRS) [13, 14], and each had neuropathological confirmation of $\mathrm{AD}$ (neuritic plaques and NFTs in numbers to meet the Consortium to Establish a Registry for Alzheimer's Disease [CERAD] criteria [15]). The interval between the last clinical examination and death generally was less than 1 year $(8.5$ months on average).

Exclusion criteria included a history of stroke, schizophrenia, motor neuron disease, presence of any central nervous system (CNS) infections, or clinical and pathological diagnosis of PD (Lewy bodies in the substantia nigra). Patients who were taking neuroleptic medication at any time were also excluded, to rule out the possibility of drug-induced EPSs.

$A D$ patients were separated into two groups, based on the presence or absence of EPSs. Significant EPSs were considered present if there was any resting tremor $(1+$ or greater $)$ or $2+$ rigidity; these ratings have been shown to be highly reliable [14]. Speech, facial expression, and bradykinesia were not included because ratings of these signs are less reliable.

CONTROL SELECTION. Control cases were obtained by sampling all autopsy cases at the medical center during the same period. Control subjects were matched to the AD patients for age and sex, and had a medical evaluation extensive enough to document general medical, neurological, and cognitive status. A neurologist (M. R. C.) reviewed each control subject's medical record to ensure that criteria for dementia and EPSs were not present for 5 years prior to death.

Brains from 11 PD and 9 PSP patients were obtained during the same years (1989-1994) and were selected from the files of the Neuropathology Unit of the Columbia Presbyterian Medical Center. Reports and slides from these cases were reviewed for accuracy of final diagnosis.

\section{Neuropathological Evaluations}

The right hemibrain was sectioned coronally and samples were taken from the substantia nigra at the level of the red nucleus and superior colliculus. In $4 \mathrm{AD}$ and 4 control brains, samples of the nigra were also taken at a more caudal level, at the point of decussation of the superior cerebellar peduncle. The basal ganglia and subthalamic nucleus were taken from the level of the anterior limb of the internal capsule and the level of the anterior thalamic nuclei separately. Sections of midfrontal, superior temporal, and inferior parietal regions of the cortex and of the hippocampus were also examined. Tissue blocks were processed through paraffin embedding. Sections were stained with hematoxylin and cosin for general screening. In addition, sections of the substantia nigra, basal ganglia, and subthalamic nucleus were stained with the Gallyas silver method and with paired helical filaments (PHFs) and ALZ-50 antibodies, all of which gave similar results, for recognizing NFTs and neuropil-like threads [16-18]. The NFT and NPT counts in the nigra were obtained in four standard $1-\mathrm{mm}^{2}$ areas (see below). The density of NPTs was determined by examining each of the four squares in the nigra and counting NPTs in the densest $10 \times$ microscope field therein. The maximal NPT density was then expressed on a four-point scale $(0=$ absent, $1=$ sparse [1-5 profiles $/ 10 \times$ microscope field], $2=$ moderate [5-15 profiles], $3=$ severe $[>15$ profiles; the photomicrograph in Fig 3 illustrates a "severe" NPT pathology]). NFT density was expressed as the average number per square in all four areas per nigra. We used an antiubiquitin antibody [19] to recognize Lewy bodies. Neither this nor hematoxylin and eosin stains revealed Lewy bodies in the nigra or locus ceruleus, basal forebrain, hypothalamus, or neocortex in any of the AD brains. NFTs and NPTs were counted over the entire sectional areas of the basal ganglia and subthalamic nucleus.

To quantitate numbers of neuronal profiles in the pars compacta of the substantia nigra, we divided the nigra into quarters, and placed a square of $1-\mathrm{mm}^{2}$ area in each quarter, similar to the analysis carried out by Rinne and colleagues [3] (Fig 1). We counted all neuronal perikarya recognizable

Fig 1. Transverse section of the substantia nigra at the level of the superior colliculus (sc) and caudal red nucleus ( $\mathrm{rn}$ ). The squares show the areas where neuronal, neurofibrillary tangle, and neuropil thread densities were studied. Ia = medial; $I b=$ midmedial; $I I$ and III = midlateral and lateral areas.

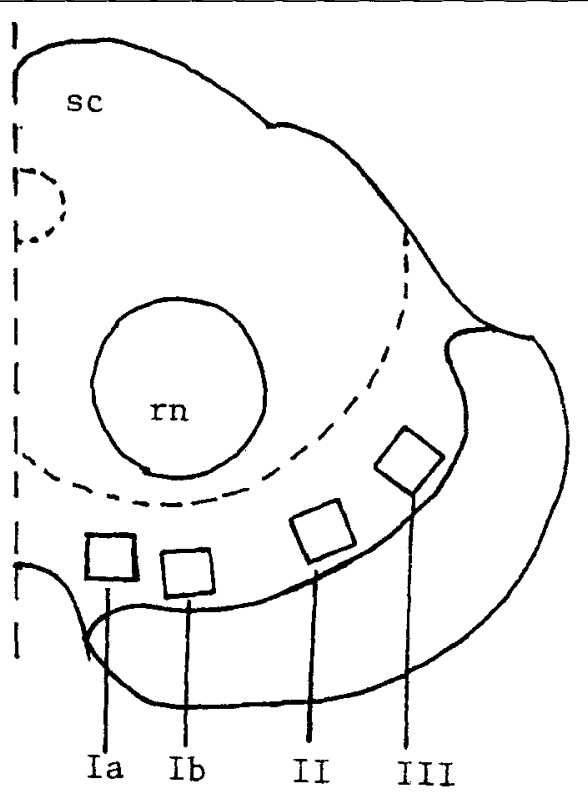


by pigment in each area. Profiles falling on grid lines were treated according to Gundersen's rule [3].

This method of counting allows one to determine the numbers of neuronal profiles in four standard areas of the nigra, yielding what is essentially a profile density. The relationship of this number to the actual numbers of nigral neurons was not determined. Change in the size of neuron profiles affects their likelihood of being counted within a section, and therefore alters the relationship between the number of counted profiles and the actual number of neurons present [20]. We therefore assessed neuron size in AD and control brains by measuring their cross-sectional area. This was done by coupling a Nikon Labophot microscope to a Dage 81 Series video camera, digitizing profiles under a $60 \times$ objective, and determining area using a Loats Associates Inquiry image analysis system.

All autopsy evaluations were based on pathological diagnostic criteria (CERAD criteria). Both neuropathologists (Y. L., J. E. G.) examined those neuronal changes in the substantia nigra.

The PD patients showed few pathological changes of the AD type (4 with NFTs only in the entorhinal cortex, layer II; only 1 with few NFTs in CAl hippocampus; none with neocortical NFTs; 10 with rare, diffuse amyloid plaques in the neocortex; and 1 with moderate, diffuse and neuritic plaques in the neocortex).

Table 1. Extrapyramidal Sign Ratings in AD Patients

\begin{tabular}{|c|c|c|c|c|c|}
\hline \multirow{2}{*}{$\begin{array}{l}\text { Case } \\
\text { No. }\end{array}$} & \multirow{2}{*}{$\begin{array}{l}\text { Age } \\
(\mathrm{yr})\end{array}$} & \multirow[b]{2}{*}{ Sex } & \multicolumn{3}{|c|}{ Extrapyramidal Sign } \\
\hline & & & Rigidity & Tremor & Bradykinesia \\
\hline 1 & 88 & $\mathrm{~F}$ & ++ & - & ++ \\
\hline 2 & 68 & $\mathrm{~F}$ & ++ & ++ & ++ \\
\hline 3 & 75 & $\mathrm{~F}$ & ++ & ++ & + \\
\hline 4 & 54 & M & ++ & ++ & + \\
\hline 5 & 59 & M & ++ & - & ++ \\
\hline 6 & 69 & $F$ & ++ & + & + \\
\hline 7 & 78 & $\mathrm{~F}$ & + & + & - \\
\hline 8 & 83 & M & ++ & - & ++ \\
\hline 9 & 86 & $\mathrm{~F}$ & $+t$ & + & ++ \\
\hline 10 & 93 & $\mathrm{~F}$ & ++ & ++ & ++ \\
\hline 11 & 75 & $\mathrm{~F}$ & ++ & ++ & ++ \\
\hline 12 & 80 & $\mathrm{~F}$ & $+t$ & ++ & ++ \\
\hline 13 & 79 & $\mathrm{M}$ & ++ & - & + \\
\hline 14 & 79 & M & ++ & - & + \\
\hline 15 & 89 & $\mathrm{~F}$ & ++ & - & + \\
\hline 16 & 89 & $\mathrm{M}$ & ++ & - & - \\
\hline 17 & 66 & $\mathrm{~F}$ & ++ & ++ & ++ \\
\hline 18 & 69 & $\mathrm{M}$ & ++ & ++ & ++ \\
\hline 19 & 69 & $\mathrm{M}$ & + & - & - \\
\hline 20 & 74 & $M$ & + & - & + \\
\hline 21 & 83 & $M$ & - & - & - \\
\hline 22 & 95 & $\mathrm{~F}$ & + & - & - \\
\hline 23 & 69 & $\mathrm{M}$ & - & - & - \\
\hline 24 & 57 & $\mathrm{~F}$ & + & - & -- \\
\hline 25 & 81 & $\mathrm{M}$ & - & $+^{a}$ & ++ \\
\hline 26 & 76 & $\mathrm{M}$ & - & - & - \\
\hline 27 & 60 & $\mathrm{M}$ & - & - & -- \\
\hline 28 & 91 & $\mathrm{~F}$ & - & - & - \\
\hline
\end{tabular}

a Sign noted on one occasion, but not on subsequent evaluations. $-=$ no signs; $+=$ slight signs; $++=$ moderate to severe signs.

\section{Data Analysis}

The differences between individual patient groups were evaluated by independent samples $t$ test or by categorical data $\chi^{2}$ statistics. Conditional logistic regression techniques for matched studies were used to estimate the association between $\mathrm{AD}$ (with $[+]$ or without $[-]$ EPS) and neuropathological findings. Analyses were controlled for age and sex. One-way analysis of variance was used to evaluate the differences in the magnitude of nigral degenerative changes among the control, $\mathrm{AD}, \mathrm{PD}$, and PSP groups.

\section{Results}

\section{Clinical Features of $A D$ Patients}

Eighteen of the 28 AD patients met criteria for the presence of EPSs (Table 1). These 18 patients had scores of moderate to severe on rigidity or tremor (AD/ EPS +). Seventeen of them displayed severe neck, limb, or postural rigidity, and 1 patient had slight rigidity and a resting tremor. No individual had an isolated rigidity of the neck, arm, or leg. Action or postural tremors were not used as EPS criteria. The 10 other patients had mild or no EPSs. One of these 10 patients had a score of 2 for bradykinesia but only had an occasional tremor. Demographic and clinical characteristics of control and AD groups are shown in Table 2. $t$ Tests revealed that $\mathrm{AD}$ (with and without EPSs) patients were significantly older than the control group ( $p=0.02$ ), but there were no differences in age, gender, or the duration of AD between the AD/EPSand AD/EPS + groups.

To determine whether EPSs occur at a late stage of $\mathrm{AD}$, we looked for associations between the Clinical Dementia Rating (CDR) score (range, $0-5$, with 5 being most severe) and the presence of EPSs. At the initial evaluation at Columbia Presbyterian Medical Center, 10 patients displayed EPSs, while 18 others did not. Eight of the 18 eventually did develop EPSs; 10 never did. At the initial evaluation, the 10 individuals with EPSs had a mean CDR score of 2.40 (scores ranging from 0-5), whereas the mean CDR score of the 8 patients without EPSs who would eventually develop EPS was 1.25 (scores ranging from $1-2)(p=0.06$ ). Of the 18 patients who had or developed EPSs, 10 had

Table 2. Age, Sex, and Clinical Variables of the AD Patients

\begin{tabular}{lllll}
\hline Groups & Age $(\mathrm{yr})^{\mathrm{a}}$ & Sex $(\mathrm{F} / \mathrm{M})$ & EPS $^{\mathrm{b}} / \mathrm{AD}$ & $\begin{array}{l}\text { Duration } \\
\text { of AD }\end{array}$ \\
\hline $\begin{array}{l}\text { Control } \\
(50)\end{array}$ & $69.1 \pm 9.8$ & $25 / 25$ & & \\
$\mathrm{AD}$ & $75.3 \pm 10.9$ & $13 / 15$ & $18 / 28$ & $6.8 \pm 3.3$ \\
$(28)$ & & & & \\
\hline
\end{tabular}

"Mean \pm standard deviation.

b According to Unified Parkinson's Disease Rating Scale (UPDRS). $\mathrm{AD}=$ Alzheimer's disease $\mathrm{F}=$ female; $\mathrm{M}=$ male; $\mathrm{EPS}=$ extrapyramidal sign. 

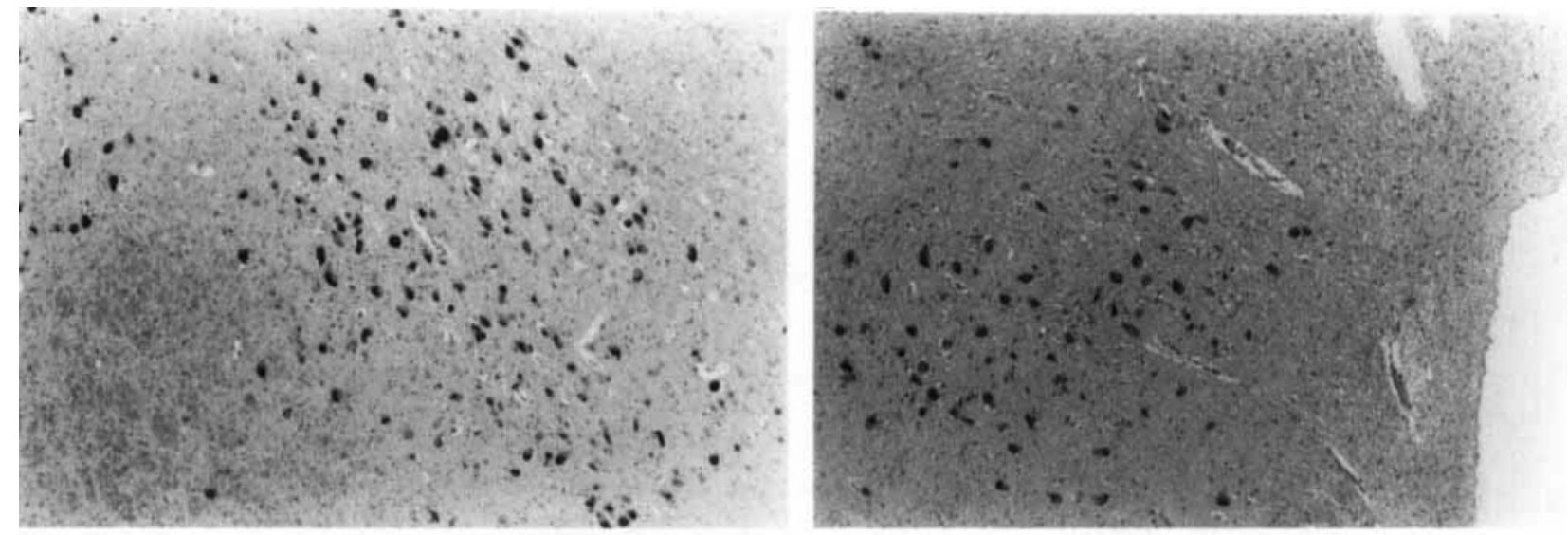

C

D
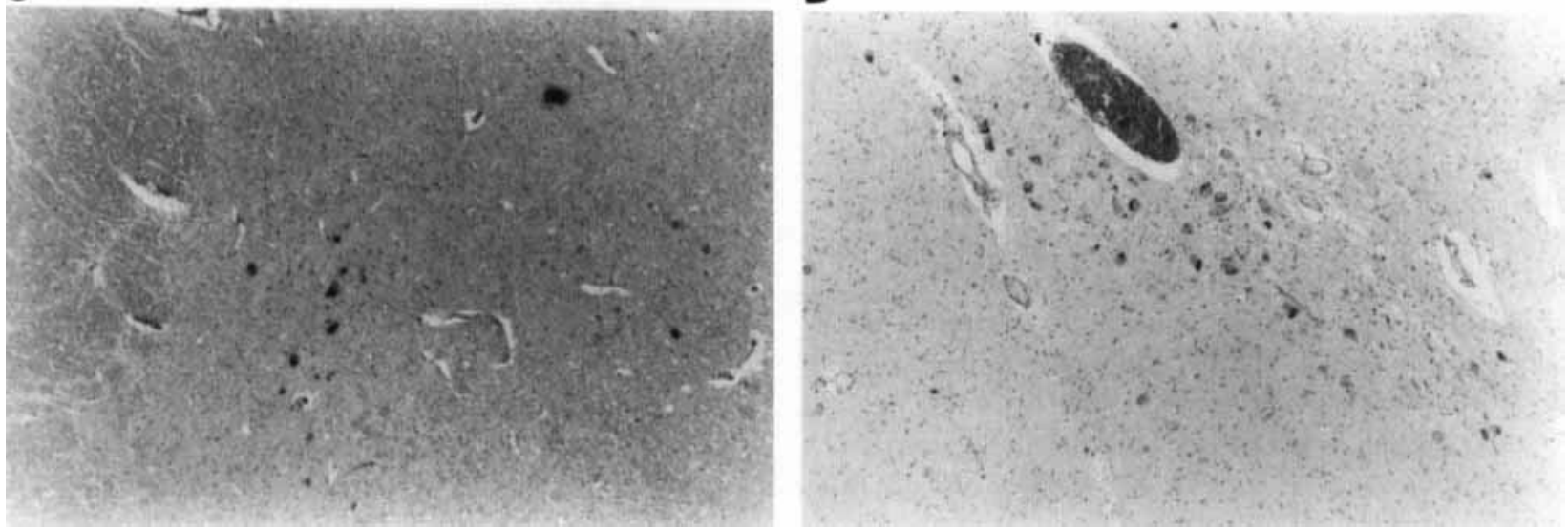

Fig 2. Representative photomicrographs of the medial (Ia) region of the substantia nigra. (A) Control. (B) AD. (C) Parkinson's disease. (D) Progressive supranuclear palsy. $(\times 80$.

CDR scores of 0 to 2 when EPSs were first found, and 8 had scores of 3 to 5 ; the average CDR score at the time when EPSs were initially observed was $2.39 \pm$ 1.24 (standard deviation [SD]). All patients but one were ambulatory at the first determination of EPS.

\section{Comparison of Nigral Degenerative Changes}

There were two comparisons in this study: The first was a comparison among $\mathrm{AD}$ patients as an entire group, the elderly control group, PD patients, and PSP patients; the second was a comparison between $\mathrm{AD} /$ EPS- and AD/EPS+ groups. The three nigral variables assayed were neuronal profile number, NFTs, and NPTs (Figs 2, 3).

We compared the numbers of neuronal profiles in control, AD, PD, and PSP brains using a one-way analysis of variance (Fig 4). The number of neuronal profiles was not significantly reduced in the $\mathrm{AD}$ group compared to the control group $(1,156.2 \pm 352.0$ $[$ mean $\pm \mathrm{SD}]$ in $\mathrm{AD}, 1,314.2 \pm 385.4$ in controls,
Fig 3. Gallyas silver staining of the substantia nigra from the brain of an AD patient with extrapyramidal signs shows neurofibrillary tangles (arrows) and many neuropil threads (arrowheads). This would correspond to a rating of 3 (severe) for neuropil threads. ( $\times 400$ before $53 \%$ reduction.)

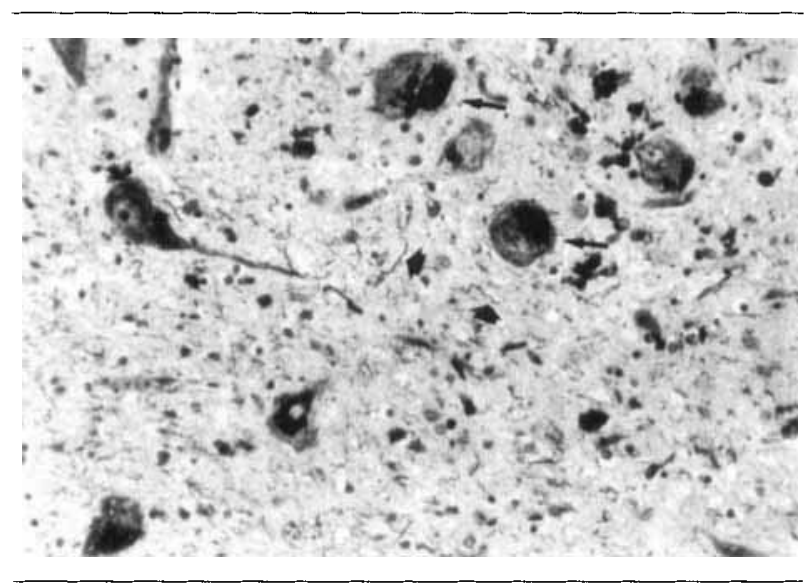




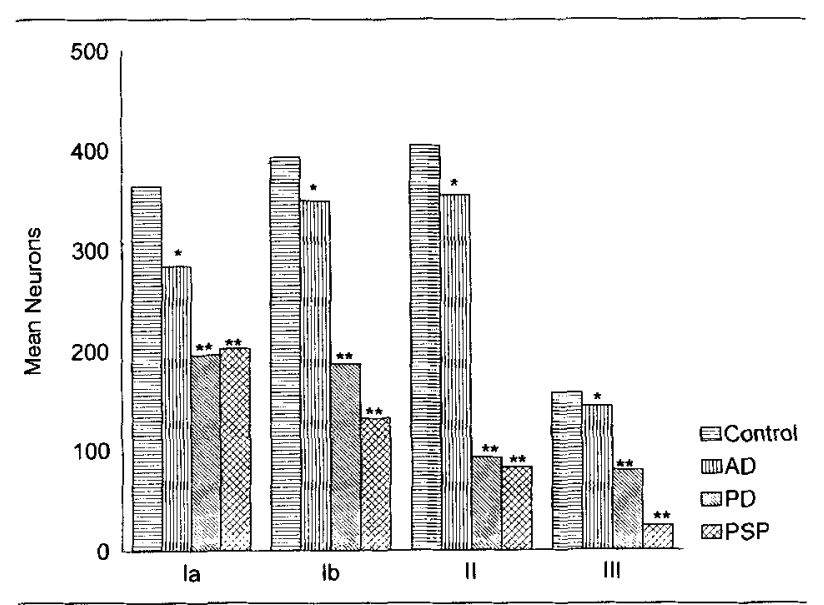

Fig 4. Analysis of numbers of nigral neurons in each of the four test areas in control, all AD, Parkinson's disease (PD), and progressive supranuclear palsy (PSP) brains. ${ }^{*} \mathrm{p}<0.5$; $*^{* *} \mathrm{p}<0.0001$.

$p=0.5$ ), but there was a severe loss in PD and PSP compared to control brains $(563.4 \pm 134.6$ in $\mathrm{PD}$, $406.6 \pm 83.4$ in PSP, $p<0.0001)$. When AD patients were separated into AD/EPS- $(n=10)$ and AD/EPS $+(n=18)$ subgroups, there was still no significant difference between the numbers of nigral neuronal profiles in $\mathrm{AD} / \mathrm{EPS}+$ patients and in controls $(p=0.4)$.

Counting neuronal profiles in the above-described manner thus revealed a $57 \%$ average decrease in PD brains, compared to control brains, similar to the $66 \%$ decrease in nigral neurons in PD brains determined stereologically [21]. As noted in the methods section, this type of counting can be biased by changes in the sizes of neurons. We do not know whether there was a significant decrease in the mean volume of nigral neurons in $\mathrm{AD}$ compared to control brains (volume measurements of pigmented nigral neurons in PD show only a modest decrease [22]). We therefore measured the cross-sectional area of pigmented neurons in control and $\mathrm{AD}$ brains, using sections that intersect the nucleolus. In analyzing 9 to 10 randomly selected neurons per nigra from 10 control and $10 \mathrm{AD}$ brains, we found no significant differences between the areas of neuronal profiles in control and AD brains (respectively, $790.7 \pm 265.1 \mathrm{\mu m}^{2}$, mean $\pm \mathrm{SD}, \mathrm{n}=103$, vs $660.5 \pm 296.0 \mu \mathrm{m}^{2}$, mean $\pm \mathrm{SD}, \mathrm{n}=94, p=0.08$ ), suggesting that size differences did not bias our profile counts.

In contrast with the normal neuronal numbers in $A D$, the entire $A D$ group showed significantly higher numbers of NFTs and NPTs than did the control group ( $p<0.0001$ ) (Fig 5). There was no difference between AD and PSP in the numbers of NFTs and NPTs. There were no NFTs in the PD nigra, but we did find NPTs, although the numbers were less than

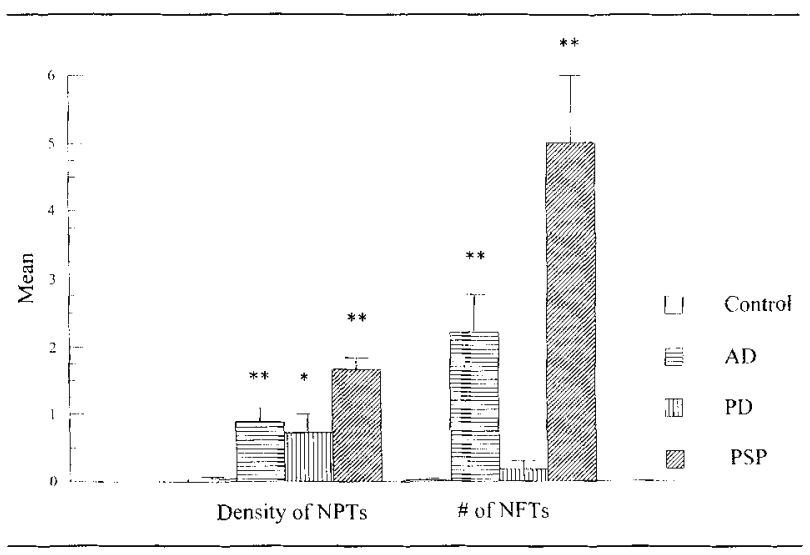

Fig 5. Analysis of nigral neurofibrillary tangles (NFTs) and neuropil threads (NPTs) in control, AD, Parkinson's disease (PD), and progressive supranuclear palsy (PSP) brains, conducted over all four test areas. Mean values \pm standard error of mean for NPT density and NFT numbers are given, calculated as described in the methods section. ${ }^{*} \mathrm{p}<0.03$; ${ }^{*} \mathrm{p}<$ 0.0001 .

those in AD nigra $(p<0.03)$. In a comparison within the $\mathrm{AD}$ group, there were significantly larger numbers of NFTs and NPTs in the AD/EPS + group than in the AD/EPS - group (for NFTs, $\chi^{2}=7.9, p<0.05$; for NPTs, $\chi^{2}=7.6, p<0.04$ ) (Fig 6).

In $4 \mathrm{AD} / \mathrm{EPS}+$ and 4 control nigras, we examined neuronal profiles, NFTs, and NPTs at a more caudal level and compared these values to those at the more rostral level in the same brain. In all brains, there were no significant differences $(p=0.25$ for neuronal profile numbers in control and $p=0.15$ for $\mathrm{AD}$ brains; $p=0.64$ for NFTs and $p=0.42$ for NPTs).

\section{Comparison of Pathological Changes in Basal} Ganglia and Subthalamic Nucleus All $(n=9)$ PSP brains showed NFTs and NPTs in the subthalamic nucleus and basal ganglia, with widespread

Fig 6. Analysis of nigral neurofibrillary tangles (NFTs) and neuropil threads (NPTs) in AD with extrapyramidal signs (AD/EPS+) and without extrapyramidal signs (AD/EPS-). Data are displayed as in Figure 5. ${ }^{*} \mathrm{p}<0.05 ;{ }^{* *} \mathrm{p}<0.04$.

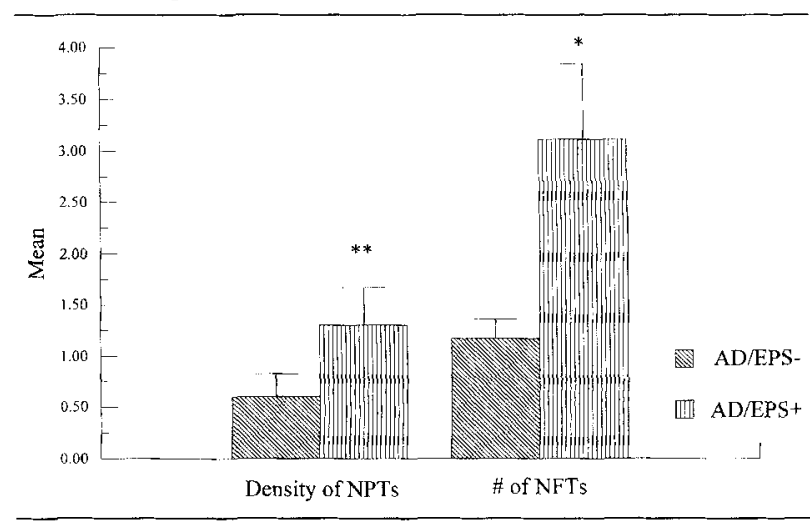


neuronal loss. There were no NFTs or NPTs in the putamen, globus pallidus, and subthalamic nucleus in control or PD brains. However, there were mild to moderate numbers of NPTs in the putamen and globus pallidus in AD/EPS + (3/18) and AD/EPS- (1/ 10) brains.

\section{Comparison of Changes in Cortex in $A D$ with and without Extrapyramidal Signs}

We examined sections of frontal, parietal, superior temporal, and striate cortex and hippocampus in all $\mathrm{AD}$ patients by semiquantitative criteria [15]. There were no differences in the numbers of amyloid plaques (neuritic or diffuse), NFTs, or NPTs between AD patients with and those without EPS.

\section{Discussion}

The frequency of nigral NFTs and NPTs was significantly higher among AD patients with EPS compared to those without EPS. These pathological changes were found in both the lateral and medial parts of the substantia nigra, suggesting that the degenerative process in the nigra affects the dopaminergic afferent parhway not only to the putamen but also to the caudate nucleus [3]. Neurons in the medial part of the nigra together with the neurons of ventral tegmental area are known to project to the limbic and cortical areas [15] and may be involved in the cognitive impairment in AD. Because we excluded patients with Lewy bodies to isolate the AD pathology from that of PD, there was no coexistent $P D$ pathology to account for the nigral degenerative changes.

The topography of degenerative changes in the substantia nigra has been studied in other neurodegenerative disorders such as PD and PSP. The loss of neurons from the substantia nigra was more conspicuous in PD and PSP than in AD. However, far more NFTs were seen in the AD brains than in the PD brains, suggesting that this nigral degenerative change is primarily involved in $\mathrm{AD}$.

The presence of EPSs in AD was not associared with neuronal loss in the substantia nigra, when compared with control cases and with the profound losses in PD and PSP. This observation is consistent with previous reports of normal nigral populations in $A D$ [5-8] and normal levels of tyrosine hydroxylase, $D_{2}$ receptors, and dopamine transporter in $\mathrm{AD}$, with and without parkinsonism [11]. Thus, the lack of cell loss in AD/ EPS + suggests that if nigral dysfunction is related to EPSs, then the surviving neurons do not function normally. The cytoskeletal pathology we observed in the $\mathrm{AD}$ nigra may well be a sign of such dysfunction. For example, altered axonal transport, either due to the cytoskeletal pathology or reflected in the cytoskeletal pathology, could in turn alter synaptic levels of molecules required for dopaminergic function, such as dopamine transporter (the loss of which was noted by Murray and coauthors [11]).

In this study, we compared nigral pathology in $A D$, $\mathrm{PD}$, and PSP brains to normal brains, paying particular attention to the changes that might correlate with EPSs. Our analysis was based on chart reviews of AD, PD, and PSP patients evaluated during life by neurologists and then a postmortem pathological study. We are not able to rule out the possibility that some of the control group did develop EPSs that were simply not detected or not mentioned. However, a pathological analysis of control cases did not show any to have developed PD, PSP, or AD.

Differences in the speed of cognitive and functional decline are important factors for prognosis in patients with AD. Reports in the literature linked faster deterioration in AD to the presence of EPSs [1,23]. Some studies $[6,9,10]$ used neuropathological methods to address this issue, but the results were inconsistent and did not relate specific AD pathology to the clinical EPSs. In this study, we used autopsy-confirmed AD cases to explore a possible association between nigral degenerative changes and EPSs in AD. The findings suggest that degenerative changes in the substantia nigra, especially NFTs and NPTs, represent correlates of EPSs.

We did not find a strong association between the CDR scales of the AD patients and the presence of EPSs or between the extent of pathology in the neocortex and substantia nigra. Thus, EPSs do not appear to be unique to the late stages of $\mathrm{AD}$. That some patients with $\mathrm{AD}$ never develop EPSs also argues that cortical pathology and nigral pathology can progress independently. Why the nigra develops pathological changes in some individuals is not clear.

This work was supported by National Institutes of Health grants AG07232, AG08702, and AG07370 and the Parkinson Disease Foundation.

We would like to thank Dr Andrew Dwork for performing the ALZ-50 immunohistochemistry staining and Drs Richard Mayeux and Robert Burke for their helpful discussions and comments.

\section{References}

1. Stern Y, Albert M, Brandt I, et al. Utility of extrapyramidal signs and psychosis as predictors of cognitive and functional decline, nursing home admission, and death in Alzheimer's disease: prospective analyses from the predictors study. Neurology 1994; $44: 2300-2307$

2. Morreil JC, Drazner M, Fulling $K$, et al. Clinical and pathological aspects of parkinsonism in Alzheimer's disease. Arch Neurol 1989;46:651-657

3. Rinne JO, Rummukainen J, Paljärvi L, Rinne UK. Dementia in Parkinson's disease is related to neuronal loss in the medial substantia nigra. Ann Neurol 1989;26:47--50

4. Mölsä PK, Säko E, Paljärvi L, et al. Alzheimer's disease: neu- 
ropathological correlates of cognitive and motor disorders. Acta Neurol Scand 1987;75:376-384

5. Ditter SM, Mirra SS. Neuropathologic and clinical features of Parkinson's disease in Alzheimer's disease patients. Neurology 1987:37:754-760

6. Leverenz J, Sumi SM. Parkinson's disease in patients with Alzheimer's disease. Arch Neurol 1986;43:662-664

7. Maslish E, Mallory M, De Teresa R, et al. Differing patterns of aberrant neuronal sprouting in Alzheimer's disease with and without Lewy bodies. Brain Res 1993;617:258-266

8. Reinikainen KJ, Paljärvi L, Halonen T, et al. Dopaminergic system and monoamine oxidase-B activity in Alzheimer's disease. Neurobiol Aging 1988;9:245-252

9. Uchihara T, Kondo H, Kosaka K, 'Tsukagoshi H. Selective loss of nigral neurons in Alzheimcr's disease: a morphometric study. Acta Neuropathol (Berl) 1992;83:271-276

10. Gibb WRG, Mountjoy CQ, Mann DMA, Lees AJ. The substantia nigra and ventral tegmental area in Alzheimer's disease and Down's syndrome. J Neurol Neurosurg Psychiatry 1989; 52:193-200

11. Murray AM, Weihmueller FB, Marshall JF, et al Damage to dopamine systems differs berween Parkinson's disease and $\mathrm{Alz}$ heimer's disease with parkinsonism. Ann Neurol 1995;37:300312

12. McKhann G, Drachman D, Folstein M, et al. Clinical diagnosis of Alzheimer's disease: report of the NINCDS-ADRDA Work Group under the auspices of Department of Health and Human Services Task Force on Alzheimer's Disease. Neurology 1984;34:939-944

13. Stern MB. The clinical characteristics of Parkinson's disease and parkinsonian syndromes: diagnosis and assessment. In: Stern $\mathrm{MB}$, Hurtig $\mathrm{Hl}$, eds. The comprehensive management of Parkinson's disease. New York: PMA, 1988:3-50
14. Richards $M$, Marder $K$, Bell $K$, et al. Interrater reliability of extrapyramidal signs in a group assessed for dementia. Arch Neurol 1991;48:1147-1149

15. Hulette C, Mirra S, Wilkinson W, et al. The Consortium to Establish a Registry for Alzheimer's Disease (CERAD). Part IX. A prospective cliniconeuropathologic study of Parkinson's features in Alzheimer's disease. Neurology 1995;45:1991-1995

16. Yen SH, Crowe A, Dickson DW. Monoclonal antibodies to Alzheimer neurofibrillary tangles. 1. Identification of polypeptides. Am J Pathol 1985;120:282-291

17. Braak E, Braak H, Mandelkow EM. A sequence of cytoskeleton changes related to the formation of neurofibrillary tangles and neuropil threads. Acta Neuropathol (Berl) 1994;87:554-567

18. Gallyas F. Silver staining of Alzheimer's neurofibrillary changes by means of physical development. Acta Morphol Acad Sci Hung 1971;19:1-8

19. Lennox G, Lowe J, Morrell R, et al. Antiubiquitin immunocytochemistry is more sensitive than conventional techniques in the detection of diffuse Lewy body disease. J Neurol Neurosurg Psychiatry $1989 ; 52: 67-71$

20. Abercrombie M. Estimation of nuclear populations from microtome sections. Anat Rec 1946;94:239-247

21. Pakkenberg B, Moller A, Gundersen HJG, et al. The absolute number of nerve cells in substantia nigra in normal subjects and in parients with Parkinson's disease estimated with an unbiased stereological method. J Neurol Neurosurg Psychiatry 1991;54: $30-33$

22. Moller A. Mean volume of pigmented neurons in the substantia nigra in Parkinson's disease. Acta Neurol Scand Suppl 1992; $137: 37-39$

23. Chui HC, Lyness SA, Sobel E, Schneider LS. Extrapyramidal signs and psychiatric symptoms predict faster cognitive decline in Alzheimer's disease. Arch Neurol 1994;51:676-681 